\title{
Sexually dimorphic effects of cannabinoid compounds on emotion and cognition
}

\section{Tiziana Rubino* and Daniela Parolaro}

Department of Structural and Functional Biology and Neuroscience Center, University of Insubria, Busto Arsizio, Italy

\author{
Edited by: \\ Viviana Trezza, University "Roma Tre," \\ Italy \\ Reviewed by: \\ Carsten Wotjak, Max Planck Institute \\ of Psychiatry, Germany \\ Antonella Gasbarri, University of \\ I'Aquila, Italy \\ Tiffany Lee, University of British \\ Columbia, Canada \\ *Correspondence: \\ Tiziana Rubino, Department of \\ Structural and Functional Biology and \\ Neuroscience Center, University of \\ Insubria, Via A. da Giussano 10, 21052 \\ Busto Arsizio, Italy. \\ e-mail: tiziana.rubino@uninsubria.it
}

This review addresses the issue of sex differences in the response to cannabinoid compounds focusing mainly on behaviors belonging to the cognitive and emotional sphere. Sexual dimorphism exists in the different components of the endocannabinoid system. Males seem to have higher CB1 receptor binding sites than females, but females seem to possess more efficient CB1 receptors. Differences between sexes have been also observed in the metabolic processing of THC, the main psychoactive ingredient of marijuana. The consistent dimorphism in the endocannabinoid system and THC metabolism may justify at least in part the different sensitivity observed between male and female animals in different behavioral paradigms concerning emotion and cognition after treatment with cannabinoid compounds. On the basis of these observations, we would like to emphasize the need of including females in basic research and to analyze results for sex differences in epidemiological studies.

Keywords: cannabinoid, sex differences, emotionality, cognition
Although the notion of sex differences in brain functionality was already present at the end of the nineteenth century (Andreano and Cahill, 2009), it is only in the last decade that increasing literature has supported and documented it. In fact for long, females were under-represented or even excluded in both clinical and preclinical studies. Indeed, until recently, the prevalent strategy in animal studies was to use males only, ironically to avoid likely sex effects.

The most characterized brain regions where functional and structural dimorphism have been studied are the hippocampus, amygdala, hypothalamus, and cortex, cerebral areas associated with cognition and emotion. Besides the anatomy, also the neurochemistry and physiology could differ in these areas between males and females. For example, dopamine, serotonin, and GABA, among others, have been shown to exhibit significant sex differences in their metabolism (Andreano and Cahill, 2009), as well as various neuropeptidergic systems (Bielsky et al., 2005; Kauffman, 2010). It is not surprising then that compounds acting in these areas and through mechanisms involving these neurotransmitters could trigger different responses in males and females.

This review addresses the issue of sex influences on the endocannabinoid system both in term of differences in the components of the system between sexes and differences in the response to cannabinoid compounds, focusing on behaviors belonging to the cognitive and emotional sphere.

\section{SEX DIFFERENCES IN THE ENDOCANNABINOID SYSTEM}

Very few data are available regarding sex differences in cannabinoid $\mathrm{CB} 1$ receptor density and coupling to $\mathrm{G}$ proteins, and fewer ones are available on the endocannabinoid levels.

Despite this limitation, a rather clear picture arises for CB1 receptor: in all the papers where $\mathrm{CB} 1$ receptor levels were measured in both male and female animals, a higher density was observed in males in almost all the cerebral regions analyzed (Rubino et al., 2008; Burston et al., 2010; Mateos et al., 2010; Riebe et al., 2010). The increase in CB1 receptor density was observed in both adolescent and adult animals, however it was stronger and wider in younger rats. For example in the adult amygdala, CB1 receptor binding site density was higher in females than males, a difference that appears to be dependent upon the presence of estradiol, since in ovariectomized female rats it was no longer seen (Riebe et al., 2010). Despite the lower receptor density, however, adolescent females showed the higher $G$ protein activation after CB1 receptor stimulation in several brain areas (Rubino et al., 2008; Burston et al., 2010), thus suggesting the presence of more efficient receptors. At adulthood, higher $\mathrm{CB} 1$ receptor/G protein coupling was still present in the prefrontal cortex of female rats (Burston et al., 2010; Mateos et al., 2010), whereas it was no longer evident in the amygdala (Mateos et al., 2010), hypothalamus, periaqueductal gray, ventral midbrain, and cerebellum (Burston et al., 2010). Contrasting data have been reported for the hippocampus: Burston et al. (2010) described higher CP-55,940-stimulated G protein activation in male rats whereas Mateos et al. (2010), reported it in females. Different hypotheses can be put forward to explain this discrepancy: first of all, different rat strains have been used, Long Evans vs. Wistar rats. A different approach was employed to assess CB1 receptor/G protein coupling, namely autoradiographic analysis on brain sections in Mateos' study and binding studies on membrane samples from brain tissue in the Burston's one. Most importantly, in the study by Mateos et al. (2010), rats underwent intense behavioral analysis before the biochemical studies whereas in that of Burston they didn't.

Sex differences in CB1 receptor density were also reported in humans, again with men showing higher binding levels in 
early adulthood (age 18-45; Van Laere et al., 2008). Sex differences were still evident later in life (age 45-70), but while men maintained or lost some CB1 binding sites, women increased them throughout the brain, thus presenting higher CB1 receptor levels at this specific interval of age (Van Laere et al., 2008).

Only one paper dealt with endocannabinoid levels in adult male and female animals (Bradshaw et al., 2006). Among the seven different brain areas analyzed, the authors found no significant differences in anandamide levels between male and female rats, whereas 2-arachidonoylglycerol (2-AG) was higher in the female pituitary gland and hypothalamus, but lower in the cerebellum. When the different phases of the estrous cycle were taken into account the picture became more complex, with fluctuation of the endocannabinoid levels among them and therefore much more diversity between male and female rats. In neonatal rats, females had lower amounts of the endocannabinoids 2-AG and anandamide in the amygdala and, accordingly, higher content of the endocannabinoid degradation enzymes, fatty acid amid hydrolase and monoacylglycerol lipase than males in this cerebral area (Krebs-Kraft et al., 2010).

\section{SEX DIFFERENCES IN THE PHARMACOKINETICS OF CANNABINOID COMPOUNDS}

Animal studies have shown sex differences in the metabolic processing of delta 9-tetrahydrocannabinol (THC). For example THC was oxidized selectively to 11-OH-delta 9-THC by liver microsomes of female rats, a form that retains the potency of THC, while in male rats, besides 11-OH-delta 9-THC, it was biotransformed to at least three different less active metabolites (Narimatsu et al., 1991). Accordingly, after intraperitoneal injections of THC, levels of its metabolites in brain tissue, including 11-OH-delta 9THC, the major active metabolite, were higher in females than in males (Tseng et al., 2004). Moreover cannabinoids are lipophilic and are sequestered in fat tissue. Adult male rats have a greater percentage of body fat than adult females and therefore their fat cells may retain more THC allowing a smaller amount to reach the brain.

\section{SEX DIFFERENCES IN THE RESPONSE TO CANNABINOID COMPOUNDS}

In view of this consistent dimorphism in the endocannabinoid system and THC metabolism, it is not surprising that cannabinoid compounds, and particularly THC, might have different effects when administered in male or female animals. Despite this obvious observation, very few studies have taken into account this possibility, performing the same experiments in both males and females. Curiously enough, most of them regarded the long-term effects of adolescent exposure to cannabinoids with particular emphasis on cognition and emotionality.

When the object recognition test was used, adolescent exposure to increasing doses of the synthetic cannabinoid agonist CP-55,940 for 21 days (post-natal days 30-50) induced impaired working memory checked following a long drug-free period in both female (O'Shea et al., 2004) and male rats (O'Shea et al., 2006). However, the same treatment at adulthood led to long-term memory impairments in male but not female rats (O'Shea et al., 2004, 2006).
In contrast, when the spatial memory was assessed through the Morris water maze, THC significantly disrupted learning in the adolescent males and females and also in adult females, whereas it did not affect learning in adult males (Cha et al., 2007). However chronic THC during either adolescence or adulthood had no effect on spatial learning in animals of both sexes tested after a long drug-free period (Cha et al., 2007). Accordingly, Higuera-Matas et al. (2009) reported that also the cannabinoid agonist CP-55,940 administered during adolescence did not affect adult performance of animals of both sexes in the water maze. In our work, both male and female rats showed spatial working memory deficits tested in the radial maze long after adolescent exposure to THC (Rubino et al., 2009a,b).

As a whole, this behavioral picture seems to suggest that whenever the exposure to cannabinoid agonists occurs during adolescence, it disrupts cognitive behaviors in both sexes if animals were tested immediately after, whilst the presence of long-term effects might depend upon the specific type of memory assessed and the sex of the animals.

Besides the behavioral picture, also the molecular underpinnings of the cognitive impairments induced by cannabinoids might present sexual dimorphism. For example we showed that THC, although inducing the same behavioral deficit in the radial maze in both male and female rats, triggered a different cellular alteration at the level of brain circuitries (Rubino et al., 2009a,b). In adult female rats exposed to THC in adolescence the spatial working memory impairment was correlated to a significant decrease in synaptophysin and PSD95 proteins in the prefrontal cortex. Moreover, proteomic analysis of the synaptosomes from this brain area, demonstrated the presence of less active synapses characterized by reduced ability in maintaining normal synaptic efficiency (Rubino et al., 2009a), thus suggesting the occurrence of altered synaptic plasticity throughout the prefrontal cortex in THC-pre-exposed female rats. In adult male rats chronically treated with THC during adolescence, the spatial working memory deficit was instead related to a significant decrease in the astroglial marker GFAP as well as in pre- and post-synaptic protein expression (VAMP2, PSD95) and NMDA receptor levels in the hippocampus. These animals also exhibited lower total dendritic length and number as well as reduced spine density in the hippocampal dentate gyrus (Rubino et al., 2009b), suggesting that male THC pre-treated rats may establish less synaptic contacts and/or less efficient synaptic connections throughout the hippocampus.

These data support the notion that males and females may use differing neural paths to reach the same behavioral end point (see for review Andreano and Cahill, 2009) and that the same THC exposure may have different neuronal consequences in the brain of male or female rats.

At the human level, besides the well-known notion that acute cannabis intoxication has been associated with transient and reversible decrements in attention, memory, and executive functions (see for review Solowij and Pesa, 2010), no evidence exists about sexual dimorphism in this dimension. Females are still too under-represented in epidemiological studies to gain a picture of different cognitive effects after THC exposure in men and women. 
Moreover, although few papers addressed this issue, they seem to support the notion that adolescent female rats appear to be more sensitive to the long-lasting effects triggered by chronic cannabinoid consumption on emotional responses than males; on the contrary, at adulthood, no sex differences are evident, or even males appear to be the more affected in the emotional domain. In fact, chronic CP-55,940 in adolescence impaired social interaction in both male and female rats, however when the same treatment was performed in adult animals, only males were affected (O'Shea et al., 2004, 2006). Moreover, when HU210 was chronically administered in adult male and female rats, a significant antidepressant response was observed in both sexes (Morrish et al., 2009). In the hole board test, which measures the propensity for novelty and uncertainty, adolescent CP-55,940 treatment increased general motor activity and inspective exploration in female rats, whereas decreased explorative behavior without affecting general motor activity in males (Biscaia et al., 2003). Finally we observed that adolescent exposure to THC triggered the development of a complex depressive-like phenotype at adulthood only in female rats, male rats not presenting both behavioral and biochemical parameters of depression (Rubino et al., 2008). Among the biochemical parameters, the transcription factor CREB seems to be involved in both the mechanism of action of antidepressants as well as the disease itself (Blendy, 2006). Accordingly, adolescent THC significantly reduced $\mathrm{pCREB}$ in the prefrontal cortex and hippocampus of female rats but not in males. Conversely, elevated CREB activity in the NAc produces various depressive-like effects in rodents (see for review Carlezon et al., 2005), and THC significantly increased it in the nucleus accumbens of female rats. Again, male rats showed no changes in pCREB levels in this cerebral region (Rubino et al., 2008).

As a whole these data seem to suggest that the adolescent female brain is more vulnerable to the adverse effects of chronic cannabinoid administration on emotional behavior than the adult brain. In support of this, when the same chronic THC treatment performed in adolescent female rats was administered in adult females, it did not induce long-lasting impairment in the emotional domain (Realini et al., 2011). The reason for this vulnerability is still unknown, however, possible sex steroid-dependent differences in the sensitivity of certain neuronal processes to cannabinoid treatment could be put forward. Accordingly, it was reported the existence of fluctuations along the ovarian cycle and sex steroid replacement in CB1 receptor density and affinity in certain brain areas (Rodríguez de Fonseca et al., 1994; Riebe et al., 2010), suggesting that estradiol may affect it. Estradiol elicits anxiolytic and antidepressant effects when injected in female rats (Fink et al., 1998; Bodo and Rissman, 2006; Walf and Frye, 2009; Romano-Torres and Fernández-Guasti, 2010). Estradiol-induced changes in emotionality are sensitive to the blockade of CB1 receptors, thus suggesting that alterations in endocannabinoid activity may contribute to estradiol's ability to modulate mood and affect (Hill et al., 2007). Therefore it could be speculated that the disruption of the endocannabinoid system homeostasis by exogenous administration of cannabinoid compounds in adolescence, a period where hormonal changes leading to sexual maturation occur, might impact emotionality in developing females.
As already observed for cognitive studies, no clear evidence exists about sexual dimorphism in emotional responses to cannabinoids at human levels. However in some epidemiological studies, although not clear stated, a gender difference might be found. For example, in a study where withdrawal symptoms after cessation of cannabis use was assessed, the symptoms formed two factors, one characterized by weakness, hypersomnia, and psychomotor retardation, and the second by anxiety, restlessness, depression, and insomnia (Hasin et al., 2008). When the authors examined the relationship of demographic characteristics to cannabis withdrawal symptoms in the full sample of frequent cannabis users, gender was associated with both the weakness symptoms and the anxiety/depression symptoms. Moreover, in an Indigenous Arnhem Land community sample, a strong association between heavy cannabis use in young people and moderate-severe depressive symptoms was found, and the rates of depression were nearly a third of females and one in six males reporting moderate-severe symptoms (Lee et al., 2008).

On the other hand, clinical data seem to indicate that the endocannabinoid system may be disturbed in affective disease, especially in females (Hill et al., 2008). Serum 2-AG content was significantly decreased in female patients diagnosed with major depression, and this decrease was correlated significantly and negatively with duration of the depressive episode (Hill et al., 2008).

Together these observations suggest the potential utility of targeting the endocannabinoid system for the treatment of affective disorders in females.

Finally, in addition to cognitive and emotional ones, other cannabinoid effects have been also shown to be sexually dimorphic. Cannabinoids are more potent and in some cases more efficacious in females than males in producing antinociception and altering movement (Craft, 2005). In Long-Evans and Lister Hooded rats, females showed a significant faster acquisition of WIN 55212-2 self-administration and maintained higher levels of responding than males, suggesting that cannabinoids might be more reinforcing for females than males (Fattore et al., 2007). Ovarian hormones might be involved in the modulation of the reinforcing effect of cannabinoids, in fact, when compared to intact females, a lower percentage of ovariectomized females acquired and maintained stable drug intake (Fattore et al., 2007).

\section{CONCLUSION}

The data here reported clearly suggest the presence of sex differences in behavioral and neurochemical responses to cannabinoid compounds. The involvement of sex steroid hormones in most of the sex differences in cannabinoid-induced behavioral effects has been already put forward and appears to be the more likely explanation (González et al., 2000; Viveros et al., 2010).

Intriguingly, a very recent work even suggested the involvement of cannabinoid signaling in the establishment of normal sex differences in the brain (Krebs-Kraft et al., 2010). The authors demonstrated that early exposure to cannabinoids masculinizes social play in females without altering this behavior in males. The likely cellular mechanism for this sexual differentiation of 
the developing brain and behavior might be the regulation of cell proliferation and cell type in the developing amygdala.

On the basis of these observations, we would like to emphasize the need of including females in basic research and to analyze results for sex differences in epidemiological studies. Moreover,

\section{REFERENCES}

Andreano, J. M., and Cahill, L. (2009). Sex influences on the neurobiology of learning and memory. Learn. Mem. 16, 248-266.

Bielsky, I. F., Hu, S. B., and Young, L. J. (2005). Sexual dimorphism in the vasopressin system: lack of an altered behavioral phenotype in female V1a receptor knockout mice. Behav. Brain Res. 164, 132-136.

Biscaia, M., Marín, S., Fernández, B., Marco, E. M., Rubio, M., Guaza, C., Ambrosio, E., and Viveros, M. P. (2003). Chronic treatment with CP 55,940 during the peri-adolescent period differentially affects the behavioural responses of male and female rats in adulthood. Psychopharmacology (Berl.) 170, 301-308.

Blendy, J. A. (2006). The role of CREB in depression and antidepressant treatment. Biol. Psychiatry 59, 1144-1150.

Bodo, C., and Rissman, E. F. (2006). New roles for estrogen receptor beta in behavior and neuroendocrinology. Front. Neuroendocrinol. 27, 217-232. Bradshaw, H. B., Rimmerman, N., Krey, J. F., and Walker, J. M. (2006). Sex and hormonal cycle differences in rat brain levels of painrelated cannabimimetic lipid mediators. Am. J. Physiol. Regul. Integr. Comp. Physiol. 291, R349-R358.

Burston, J. J., Wiley, J. L., Craig, A. A., Selley, D. E., and Sim-Selley, L. J. (2010). Regional enhancement of cannabinoid CB1 receptor desensitization in female adolescent rats following repeated deltatetrahydrocannabinol exposure. $\mathrm{Br}$. J. Pharmacol. 161, 103-112.

Carlezon, W. A. Jr., Duman, R. S., and Nestler, E. J. (2005). The many faces of CREB. Trends Neurosci. 28, 436-445.

Cha, Y. M., Jones, K. H., Kuhn, C. M., Wilson, W. A., and Swartzwelder, H. S. (2007). Sex differences in the effects of delta9tetrahydrocannabinol on spatial learning in adolescent and adult rats. Behav. Pharmacol. 18, 563-569.

Craft, R. M. (2005). Sex differences in behavioral effects of cannabinoids. Life Sci. 77, 2471-2478.

Fattore, L., Spano, M. S., Altea, S., Angius, F., Fadda, P., and Fratta, W. (2007). Cannabinoid self-administration in rats: sex differences and the influence of ovarian function. Br. J. Pharmacol. 152, 795-804.

Fink, G., Sumner, B. E., McQueen, J. K., Wilson, H., and Rosie, R. (1998). Sex steroid control of mood, mental state and memory. Clin. Exp. Pharmacol. Physiol. 25, 764-775.

González, S., Bisogno, T., Wenger, T., dero, F., Di Marzo, V., Ramos, J. A., and Fernández-Ruiz, J. J. (2000). Sex steroid influence on cannabinoid $\mathrm{CB}(1)$ receptor mRNA and endocannabinoid levels in the anterior pituitary gland. Biochem. Biophys. Res. Commun. 270, 260-266.

Hasin, D. S., Keyes, K. M., Alderson, D., Wang, S., Aharonovich, E., and Grant, B. F. (2008). Cannabis withdrawal in the United States: results from NESARC. J. Clin. Psychiatry 69, 1354-1363.

Higuera-Matas, A., Botreau, F., Miguéns, M., Del Olmo, N., Borcel, E., Pérez-Alvarez, L., GarcíaLecumberri, C., and Ambrosio, E. (2009). Chronic periadolescent cannabinoid treatment enhances adult hippocampal PSA-NCAM expression in male Wistar rats but learning and memory. Pharmacol. Biochem. Behav. 93, 482-490.

Hill, M. N., Karacabeyli, E. S., and Gorzalka, B. B. (2007). Estrogen recruits the endocannabinoid system to modulate emotionality. Psychoneuroendocrinology 32, 350-357.

Hill, M. N., Miller, G. E., Ho, W. S., Gorzalka, B. B., and Hillard, C. J. (2008). Serum endocannabinoid content is altered in females with depressive disorders: a preliminary report. Pharmacopsychiatry 41, 48-53.

Kauffman, A. S. (2010). Gonadal and nongonadal regulation of sex differences in hypothalamic Kiss 1 neurons. J. Neuroendocrinol. 22, 682-691. C. J., and McCarthy, M. M. (2010). Sex difference in cell proliferation in developing rat amygdala mediated by endocannabinoids has implications for social behavior. Proc. Natl. Acad. Sci. U.S.A. 107, 20535-20540.

Lee, K. S., Clough, A. R., Jaragba, M. J., Conigrave, K. M., and Patton, Manzanares, J., Milone, A., Berrenonly has marginal effects on anxiety,

Krebs-Kraft, D. L., Hill, M. N., Hillard,

when acute cannabinoid effects are taken into account it would be very useful also to discriminate among the different female hormonal status. As a whole these data will help to better understand the therapeutic possibilities of the endocannabinoid system and to better exploit them, perhaps in a sex-dependent manner.

G. C. (2008). Heavy cannabis use and depressive symptoms in three aboriginal communities in Arnhem Land, Northern Territory. Med. J. Aust. 188, 605-608.

Mateos, B., Borcel, E., Loriga, R., Luesu, W., Bini, V., Llorente, R., Castelli, M., and Viveros, M. P. (2010). Adolescent exposure to nicotine and/or the cannabinoid agonist $\mathrm{CP}$ 55,940 induces gender-dependent long-lasting memory impairments and changes in brain nicotinic and CB1 cannabinoid receptors. J. Psychopharmacol. (Oxford). doi: 10.1177/0269881110370503. [Epub ahead of print].

Morrish, A. C., Hill, M. N., Riebe, C. J. N., and Gorzalka, B. B. (2009). Protracted cannabinoid administration elicits antidepressant behavioral responses in rats: role of gender and noradrenergic transmission. Physiol. Behav. 98, 118-124.

Narimatsu, S., Watanabe, K., Yamamoto, I., and Yoshimura, H. (1991). Sex difference in the oxidative metabolism of delta 9-tetrahydrocannabinol in the rat. Biochem. Pharmacol. 41, 1187-1194.

O'Shea, M., McGregor, I. S., and Mallet, P. E. (2006). Repeated cannabinoid exposure during perinatal, adolescent or early adult ages produces similar long lasting deficits in object recognition and reduced social interaction in rats. J. Psychopharmacol. (Oxford) 20, 611-621.

O'Shea, M., Singh, M. E., McGregor, I. S., and Mallet, P. E. (2004). Chronic cannabinoid exposure produces lasting memory impairment and increased anxiety in adolescent but not adult rats. J. Psychopharmacol. (Oxford) 18, 502-508.

Realini, N., Vigano, D., Guidali, C., Zamberletti, E., Rubino, T., and Parolaro, D. (2011). Chronic URB597 treatment at adulthood reverted most depressive-like symptoms induced by adolescent exposure to THC in female rats. Neuropharmacology 60, 235-243.

Riebe, C. J., Hill, M. N., Lee, T. T., Hillard, C. J., and Gorzalka, B. B. (2010). Estrogenic regulation of limbic cannabinoid receptor binding. Psychoneuroendocrinology 35, 1265-1269.
Rodríguez de Fonseca, F., Cebeira, M., Ramos, J. A., Martín, M., and Fernández-Ruiz, J. J. (1994). Cannabinoid receptors in rat brain areas: sexual differences, fluctuations during estrous cycle and changes after gonadectomy and sex steroid replacement. Life Sci. 54, 159-170.

Romano-Torres, M., and FernándezGuasti, A. (2010). Estradiol valerate elicits antidepressantlike effects in middle-aged female rats under chronic mild stress. Behav. Pharmacol. 21, 104-111.

Rubino, T., Realini, N., Braida, D., Alberio, T., Capurro, V., Viganò, D., Guidali, C., Sala, M., Fasano, M., and Parolaro, D. (2009a). The depressive phenotype induced in adult female rats by adolescent exposure to THC is associated with cognitive impairment and altered neuroplasticity in the prefrontal cortex. Neurotox. Res. 15, 291-302.

Rubino, T., Realini, N., Braida, D., Guidi, S., Capurro, V., Viganò, D., Guidali, C., Pinter, M., Sala, M., Bartesaghi, R., and Parolaro, D. (2009b). Changes in hippocampal morphology and neuroplasticity induced by adolescent THC treatment are associated with cognitive impairment in adulthood. Hippocampus 19, 763-772.

Rubino, T., Vigano, D., Realini, N., Guidali, C., Braida, D., Capurro, V., Castiglioni, C., Cherubino, F., Romualdi, P., Candeletti, S., Sala, M., and Parolaro, D. (2008). Chronic delta 9-tetrahydrocannabinol during adolescence provokes sex-dependent changes in the emotional profile in adult rats: behavioral and biochemical correlates. Neuropsychopharmacology 33, 2760-2771.

Solowij, N., and Pesa, N. (2010). Cognitive abnormalities and cannabis use. Rev. Bras. Psiquiatr. 32(Suppl. 1), S31-S40.

Tseng, A. H., Harding, J. W., and Craft, R. M. (2004). Pharmacokinetic factors in sex differences in delta 9-tetrahydrocannabinolinduced behavioral effects in rats. Behav. Brain Res. 154, 77-83.

Van Laere, K., Goffin, K., Casteels, C., Dupont, P., Mortelmans, L., 
de Hoon, J., and Bormans, G. (2008). Gender-dependent increases with healthy aging of the human cerebral cannabinoid-type 1 receptor binding using [(18)F]MK-9470 PET. Neuroimage 39, 1533-1541.

Viveros, M. P., Marco, E. M., LópezGallardo, M., Garcia-Segura, L. M., and Wagner, E. J. (2010). Framework for sex differences in adolescent neurobiology: a focus on cannabinoids. Neurosci. Biobehav. Rev. 35, 1740-1751.

Walf, A. A., and Frye, C. A. (2009). Effects of two estradiol regimens on anxiety and depressive behaviors and trophic effects in peripheral tissues in a rodent model. Gend. Med. 6, 300-311.

Conflict of Interest Statement: The authors declare that the research was conducted in the absence of any commercial or financial relationships that could be construed as a potential conflict of interest.

Received: 14 June 2011; paper pending published: 18 July 2011; accepted: 11 September 2011; published online: 28 September 2011.

Citation: Rubino $T$ and Parolaro $D$ (2011) Sexually dimorphic effects of cannabinoid compounds on emotion and cognition. Front. Behav. Neurosci. 5:64. doi: 10.3389/fnbeh.2011.00064

Copyright (C) 2011 Rubino and Parolaro. This is an open-access article subject to a non-exclusive license between the authors and Frontiers Media SA, which permits use, distribution and reproduction in other forums, provided the original authors and source are credited and other Frontiers conditions are complied with. 